\title{
ACTITUDES Y CONOCIMIENTOS SEXUALES DIRECTAMENTE RELACIONADOS CON LA MENSTRUACION Y SUS CREENCIAS, EN 8 COLEGIOS FEMENINOS DE POPAYAN
}

\author{
Dr. José Vicente Erazo, M.D.* \\ Dr. Braulio Lara Alvarez, M.D., M.S.P.**
}

\section{Introducción}

Parte indispensable de un curso de educación sexual es el conocimiento del público con que se va a trabajar. En nuestras culturas donde todo lo tocante al sexo está rodeado de cierto tabú es imprescindible limar las asperezas que se presentan haciendo sondeos previos para determinar el grado y tipo de información recibida a fin de orientar de manera adecuada la instrucción. Por esta razón y con el único afán de fomentar esta inquietud e iniciar algunos cursos tentativos relacionados con diversos aspectos de la sexualidad humana, y teniendo en cuenta las barreras que se presentan por parte de las directivas educacionales de los planteles que traten estos temas a manera de estrategia y vencer resistencias, crear inquietudes $y$ hacer sentir la necesidad por parte de personal docente y discente, se elaboró un sencillo cuestionario con las preguntas que revelen los conocimientos mínimos necesarios para todo adolescente.

Este pequeño recurso allanó el camino y abrió las puertas para encontrar un grupo, ansioso e inquieto por adquirir la información necesaria e indispensable para la formación de la personalidad.
Como este cuestionario estaba dirigido a personal exclusivamente femenino se orientó a los conocimienots relativos a la menstruación y sus creencias.

\section{Objetivos}

a) Conocer el grado y tipo de educación que en la actualidad se está proporcionando a la mujer en la etapa de la pubertad en relación con la menarca y ciclos menstruales.

b) Investigar las tendencias y creencias de tipo popular o folclórico que se tejen alrededor de la menstruación.

c) Averiguar la necesidad por ellos sentida, de información sobre aspectos sexuales y la manera de satisfacerla con cursillos posteriores.

\section{Metodología}

La forma mejor para cumplir a cabalidad los objetivos propuestos, era una encuesta aplicada a todos los colegios femeninos de la ciudad de Popayán; ante la imposibilidad de tomar

\footnotetext{
* Departamento de Obstetricia y Ginecología. * Departamento de Medicina Preventiva.
} 
el total del alumnado, se aplicó el formulario a las alumnas de último año de los colegios: Gabriela Mistral, San José de Tarbes, Franciscanas, Sagrado Corazón de Jesús, Centro del Inmaculado Corazón de María, SENA y Centro Colombo Americano, con un total de 229 alumnas, muestra que estimamos lo suficientemente representativa de los colegios femeninos de la ciudad.

El formulario fue aplicado por los miembros del Comité de Educación Sexual, previa estandarización de conceptos, aplicación que se hizo antes de la presentación de una película alusiva al tema que sirvió como pretexto para ganar la confianza de todo el alumnado. La película a su vez llenaba vacíos, resolvía dudas y acrecentaba conocimientos.

El tiempo necesario para diligenciar el formulario se estimó en 30 minutos, tiempo que no fue sobre fondo por ninguna de las alumnas que lo respondieron.

\section{Resulfados}

Los resultados están consignados en los 18 cuadros y las 6 gráficas mencionadas a continuación.

\section{CUADRO N: 1}

GRUPOS DE EDAD EN 229 ESTUDIANTES ENCUESTADAS EN 7 COLEGIOS FEMENINOS DE POPAYAN

\begin{tabular}{lcc}
\hline Eded en años & Número & Porcentaje \\
\hline - de 20 años & 153 & 76.49 \\
+ de 20 años & 20 & 10.23 \\
Sin especificar* & 26 & 13.49 \\
\cline { 2 - 2 } & 229 & 100.00 \\
\hline
\end{tabular}

* El grupo sin especificar es una muestra fehaciente de la tendencia existente en el sexo femenino de ocultar la edad.

\section{CUADRO NN: 2}

GRADO DE INSTRUCCION EN 229 ESTUDIANTES ENCUESTADAS EN 7 COLEGIOS FEMENINOS DE POPAYAN

\begin{tabular}{lcc}
\hline Grado de Instrucción & Número & Porcentaje \\
\hline Bachillerato & 136 & 59 \\
Comercio & 64 & 27 \\
Otros* & 29 & 14 \\
TOTAL & 229 & 100.00 \\
\hline
\end{tabular}

* Incluye cursos de capacitación del SENA.

\section{GRAFICA NN 1}

GRADO DE INSTRUCCION EN 229 ESTUDIANTES ENCUESTADAS EN COLEGIOS FEMENINOS DE POPAYAN

PORCENTAJES

\begin{tabular}{|c|c|c|c|c|c|}
\hline & 0 & 25 & 50 & 75 & 100 \\
\hline Bachillerato & & & 59.0 & & \\
\hline Comercio & & 27.0 & & & \\
\hline Otros & 14.0 & & & & \\
\hline
\end{tabular}

\section{CUADRO N: 3}

MECANISMO DE PRODUCCION DE LA MENSTRUACION SEGUN RESPUESTA DE 229 ESTUDIANTES ENCUESTADAS EN 7 COLEGIOS FEMENINOS DE POPAYAN

\begin{tabular}{lcc}
\hline $\begin{array}{l}\text { Conocimiento sobre } \\
\text { mecanismo de producción }\end{array}$ & Número & Porcentaje \\
\hline Correcto & 0 & 0 \\
Incorrecto & 101 & 44.10 \\
Sin contestar & 128 & 55.90 \\
TOTAL & 229 & 100.00 \\
\hline
\end{tabular}

Este cuadro llama la atención que a pesar del alto grado de instrucción 
de las encuestadas ( $59 \%$ son bachilleres) no se les enseñó o no asimilaron este concepto fundamental, elemental, indispensable en la formación de la mujer, puesto que el $44.10 \%$ fue incorrecto y el $59.90 \%$ no dieron información alguna por temor o manifestar su verdadera ignorancia.

\section{CUADRO NN 4}

EDAD DE APARICION Y DESAPARICION DE LA MENSTRUACION SEGUN RESPUESTAS DE 229 ESTUDIANTES ENCUESTADAS EN 7 COLEGIOS FEMENINOS DE POPAYAN

\begin{tabular}{lcr}
\hline $\begin{array}{l}\text { Edad de aparición } \\
\boldsymbol{y} \text { desaparición }\end{array}$ & Número & Porcentaje \\
\hline Correcto & 59 & 25.76 \\
Incorrecto & 57 & 24.89 \\
Sin contestar & 7 & 3.06 \\
Incompleto & 106 & 46.29 \\
\cline { 2 - 3 } TOTAL & 229 & 100.00 \\
\hline
\end{tabular}

Es de notar que en el Cuadro No 4, que en tópicos directamente rela- cionados con el sexo femenino y su fisiología las $3 / 4$ partes de las encuestadas tienen conceptos completamente erróneos.

\section{CUADRO N: 6}

CONCEPTO SOBRE ALIMENTOS QUE DEBEN EVITARSE DURANTE LA MENSTRUACION SEGUN RESPUESTAS DE 229 ESTUDIANTES ENCUESTADAS EN 7 COLEGIOS FEMENINOS DE POPAYAN

\begin{tabular}{lrr}
\hline $\begin{array}{l}\text { Alimentos que deben } \\
\text { evitarse }\end{array}$ & Número & Porcentaje \\
\hline Correcto & 65 & 28.38 \\
Incorrecto* & 158 & 69.00 \\
Sin contestar & 4 & 1.75 \\
Incompleto & 2 & 0.87 \\
TOTAL & 229 & 100.00 \\
\hline
\end{tabular}

* Incorrecto incluye aquí todas las respuestas que creen que durante el proceso menstrual se deben evitar determinados alimentos bien sea porque aumentan la hemorragia, producen dolor - alteran otras funciones orgánicas y dejan secuelas permanentes de difícil curación.

\section{CUADRO N: 5}

ACTIVIDADES QUE DEBEN EVITARSE DURANTE LA MENSTRUACION, SEGUN CREENCIAS DE 229 ESTUDIANTES ENCUESTADAS EN 7 COLEGIOS FEMENINOS DE POPAYAN

\begin{tabular}{|c|c|c|c|}
\hline Actividades & Razón para no realizarlas & Total & Porcentaje \\
\hline Ejercicios fuertes & $\begin{array}{l}\text { Produce hemorragias. Trae problemas para } \\
\text { los hijos. Provocan cólicos. }\end{array}$ & 150 & 70.53 \\
\hline Montar a caballo & $\begin{array}{l}\text { Se pierde la virginidad. } \\
\text { Produce hemorragias. }\end{array}$ & 35 & 14.49 \\
\hline Bañarse & $\begin{array}{l}\text { Perjudicial para el organismo. } \\
\text { Produce hemorragias. }\end{array}$ & 16 & 5.31 \\
\hline Contacto sexual & $\begin{array}{l}\text { Contacto sexual antihigiénico. } \\
\text { Perjudica el cuerpo. }\end{array}$ & 14 & 5.31 \\
\hline $\begin{array}{l}\text { Montar en bicicleta } \\
\text { Bailar }\end{array}$ & $\begin{array}{l}\text { Aumenta la hemorragia. } \\
\text { Aumenta la hemorragia. } \\
\text { Produce malestar y cólicos. }\end{array}$ & $\begin{array}{c}10 \\
1\end{array}$ & $\begin{array}{l}2.55 \\
0.48\end{array}$ \\
\hline Bebidas alcohólicas & Dan mal olor a la sangre menstrual. & 1 & 0.48 \\
\hline TOTAL & & 229 & 100.00 \\
\hline
\end{tabular}


Aunque la actividad física no tiene ninguna relación con el flujo menstrual se aprecia el hecho notorio de considerar la menstruación como una

GRAFICA № 2

ALIMENTOS QUE DEBEN EVITARSE DURANTE LA MENSTRUACION SEGUN RESPUESTAS DE 229 ESTUDIANTES ENCUESTADAS EN 7 COLEGIOS FEMENINOS DE POPAYAN

PORCENTAJES

\begin{tabular}{|c|c|c|c|c|c|}
\hline & 0 & 25 & 50 & 75 & 100 \\
\hline Correcta & 28. & & & & \\
\hline Incorrecta & & & 69.00 & & \\
\hline Sin contestar & & 1.75 & & & \\
\hline Incompleta & & 0.87 & & & \\
\hline
\end{tabular}

"Enfermedad" que produce incapacidad física parcial que obliga a guardar cuarentena.

\section{CUADRO № 8}

CONCEPTO SOBRE ACEPTACION DEL BAÑO DURANTE LA MENSTRUACION EN 229 ESTUDIANTES ENCUESTADAS EN 7 COLEGIOS FEMENINOS DE POPAYAN

\begin{tabular}{lcc}
\hline Aceptación del baño & Número & Porcentaje \\
\hline Correcto & 141 & 61.57 \\
Incorrecto & 58 & 25.33 \\
Sin contestar & 30 & 13.10 \\
TOTAL & 229 & 100.00 \\
\hline
\end{tabular}

Obsérvese cómo la cuarta parte de las encuestadas se privan de esta práctica higiénica durante la menstruación por creerla perjudicial en alto grado para la salud.

\section{CUADRO N: 7 \\ RAZONES POR LAS CUALES SE DEBEN EVITAR DETERNINADOS ALIMENTOS DURANTE LA MENSTRUACION. ASPECTOS FOLCLORICOS SEGUN RESPUESTAS DE 229 ESTUDIANTES ENCUESTADAS EN 7 COLEGIOS FEMENINOS DE POPAYAN}

\begin{tabular}{|c|c|c|c|}
\hline Alimentos & Por qué se deben evitar & Total & Porcentaje \\
\hline $\begin{array}{l}\text { Frutas ácidas } \\
\text { Leche }\end{array}$ & $\begin{array}{l}\text { Dan mal olor. Producen flujos. } \\
\text { Dá mal olor al flujo menstrual y origina }\end{array}$ & 143 & 59.34 \\
\hline & flujos "blancos" vaginales. & 57 & 23.65 \\
\hline Alcohol & Corta la sangre menstrual. & 9 & 3.73 \\
\hline Aguacate, panela, & Producen cólicos menstruales & & \\
\hline sustancias picantes, helados. & - aumentan la menstruación. & 20 & 8.29 \\
\hline $\begin{array}{l}\text { Fumar } \\
\text { Comidas pesadas } \\
\text { (Alimentos grasos }\end{array}$ & Produce cefálea. & 1 & 0.41 \\
\hline $\begin{array}{l}\text { o "trasnochados") } \\
\text { Todos los alimentos }\end{array}$ & Espesan la sangre. & 6 & 2.47 \\
\hline se deben evitar & Aumentan el dolor. & 2 & 0.83 \\
\hline Alimentos fríos o calientes & Producen cólicos difíciles de curar. & 3 & 1.24 \\
\hline TOTAL & & 241 & 100.00 \\
\hline
\end{tabular}

NOTA: El total de respuestas es mayor que el número de encuestadas porque muchas dieron dos respuestas. 


\section{CUADRO N: 9}

\section{CONCEPTO SOBRE LA NECESIDAD DE LA MENSTRUACION EN 229 ESTUDIANTES ENCUESTADAS EN 7 COLEGIOS FEMENINOS DE POPAYAN.}

(El $100 \%$ de las encuesíadas consideró la menstruación necesaria e indispensable para la salud, en cuanto a la razón de esa necesidad los conceptos fueron variados y se resumen a continuación)?

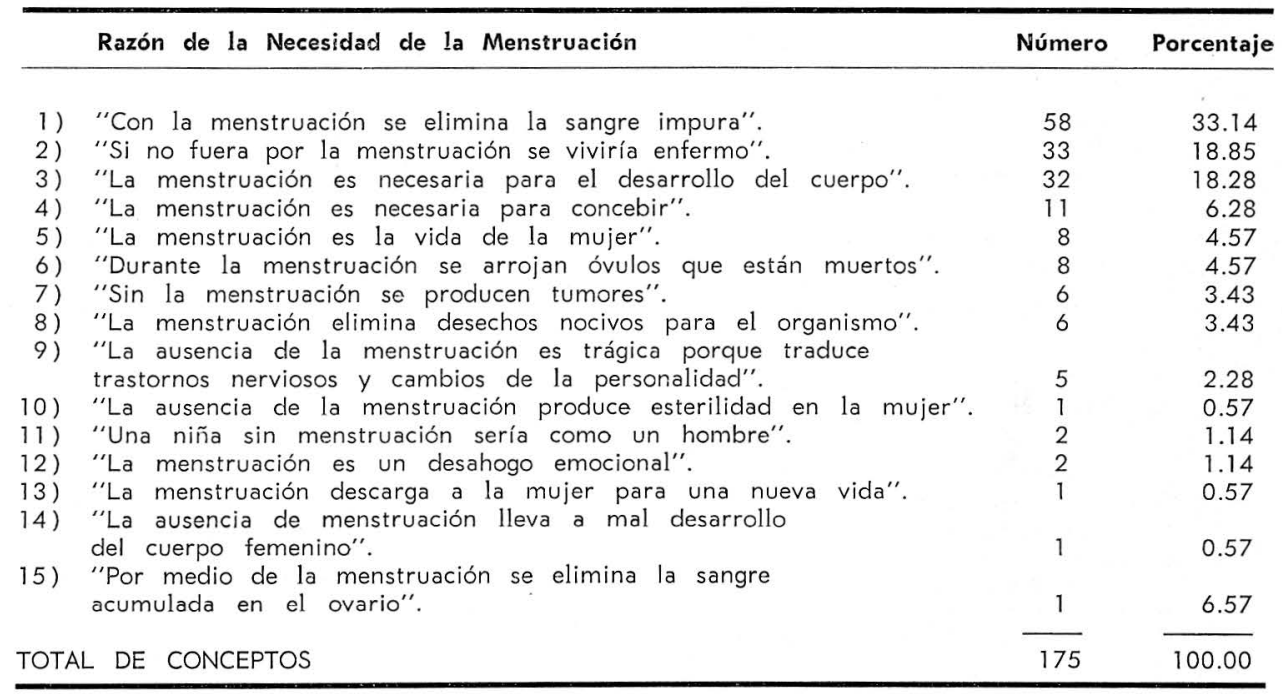

GRAFICA № 3

CONCEPTO SOBRE ACEPTACION DEL BAÑO DURANTE LA MENSTRUACION EN 229 ESTUDIANTES ENCUESTADAS EN 7 COLEGIOS FEMENINOS DE POPAYAN

PORCENTAJES

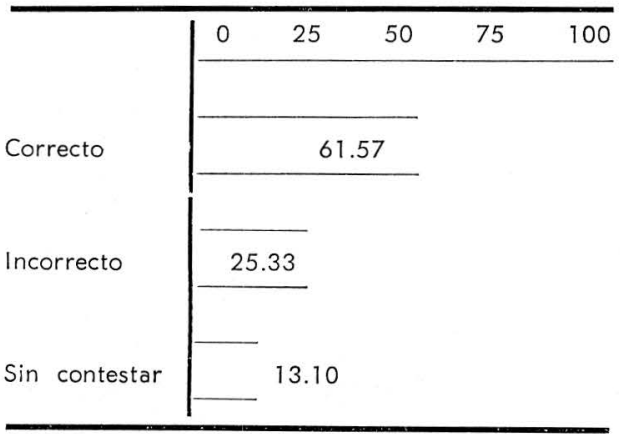

\section{CUADRO № 10}

CONC5PTO SOBR5 LA POSIBILIDAD DE EMBARAZO DURANTE LA MENSTRUACION SEGUN RESPUESTAS DE 229 ESTUDIANTES ENCUESTADAS EN 7 COLEGIOS FEMENINOS DE POPAYAN

\begin{tabular}{lrr}
\hline Posibilidad de embarazo & Número & Porcentaje \\
\hline Correcto & 117 & 51.09 \\
Incorrecto & 70 & 30.56 \\
Sin contestar & 42 & 18.34 \\
\cline { 2 - 3 } TOTAL & 229 & 100.00 \\
\hline
\end{tabular}

En este cuadro llama la atención el alto porcentaje de conceptos incorrectos y sin contestar que evidencian la ignorancia crasa en relación con la fecundación durante la menstruación, máxime que hubo conceptos de que cuando la fecundación ocurría el hijo nacería deforme. 
CUADRO N: 11

CONCEPTO SOBRE ACEPTACION DEL DEPORTE $Y$ LA NATACION DURANTE LA MENSTRUACION SEGUN RESPUESTAS DE 229 ESTUDIANTES ENCUESTADAS EN 7 COLEGIOS FEMENINOS DE POPAYAN

\begin{tabular}{lcc}
\hline Aceptación del deporte & Número & Porcentaje \\
\hline Correcto & 92 & 40.17 \\
Incorrecto & 113 & 49.33 \\
Sin contestar & 23 & 10.48 \\
\cline { 2 - 2 } TOTAL & 229 & 100.00 \\
\hline
\end{tabular}

Este cuadro evidencia el hecho comúnmente aceptado de que la mujer debe privarse de todo ejercicio durante la menstruación por considerarla como una enfermedad que como tal debe ser sometida a cuarentena.

\section{CUADRO № 12}

NECESIDAD DE DOLOR O MOLESTIAS DURANTE LA MENSTRUACION SEGUN RESPUESTAS DE 229 ESTUDIANTES ENCUESTADAS EN 7 COLEGIOS FEMENINOS DE POPAYAN

\begin{tabular}{lrr}
\hline Corcepto de dolor & Número & Porcentaje \\
\hline Necesario & 150 & 65.50 \\
No necesario & 61 & 26.63 \\
Sin contestar & 18 & 7.86 \\
TOTAL & 229 & 100.00 \\
\hline
\end{tabular}

La falta de una explicación científica adecuada sobre el mecanismo de la menstruación justifica el alto porcentaje $(65.50 \%)$ casi $2 / 3$ partes de las mujeres que asocian este proceso fisiológico con el dolor.

\section{CUADRO N: 13}

CAMBIOS DE CARACTER DURANTE LA MENSTRUACION SEGUN RESPUESTAS DE 229 ESTU.

DIANTES ENCUESTADAS EN 7 COLEGIOS FEM5NINOS DE POPAYAN

\begin{tabular}{lrr}
\hline Cambio de carácter & Número & Porcentaje \\
\hline Sin cambio & 32 & 18.34 \\
Con cambio & 173 & 75.98 \\
No contestaron & 13 & 5.67 \\
TOTAL & 229 & $\overline{100.00}$ \\
\hline
\end{tabular}

La hisioria y este gran porcentaje 75.98 nos demuestra el hecho de que la mujer sea víctima de trastornos tanto físicos como síquicos durante la época menstrual siendo esta una herencia para la mujer moderna consecuencial a la mala instrucción recibida en estos aspectos.

\section{CUADRO NN 14}

CONCEPTO SOBRE LA NECESIDAD DE LOS OVARIOS EN LA NEENSTRUACION SEGUN RESPUES TAS DE 229 ESTUDIANTES ENCUESTADAS EN 7 COLEGIOS FEMENINOS DE POPAYAN

\begin{tabular}{lrr}
\hline Necesidad de los ovarios & Número & Porcentaje \\
\hline Correcto & 209 & 90.83 \\
Incorrecto & 6 & 3.83 \\
Sin contestar & 12 & 5.24 \\
TOTAL & 229 & 100.00 \\
\hline
\end{tabular}

\section{CUADRO N: 15}

CONCEPTO SOBRE OVULACION Y EPOCA EN QUE SE PRODUCE SEGUN RESPUESTA DE 229 ESTUDIANTES ENCUESTADAS EN 7 COLEGIOS FEMENINOS DE POPAYAN

\begin{tabular}{lcc}
\hline Concepto de ovulación & Número & Porcentaje \\
\hline Correcto & 0 & 0 \\
Incorrecto & 90 & 39.30 \\
Sin contestar & 118 & 51.53 \\
Incompleto & 18 & 7.86 \\
TOTAL & 229 & 100.00 \\
\hline
\end{tabular}

Es de notar que a pesar del alto grado de instrucción los conceptos elementales sobre reproducción están vetados en algunos colegios posiblemente por considerarse "pecaminoso" lo cual explica el 0 (cero) de respuestas correctas. 


\section{GRAFICA N: 4}

CONCEPTO SOBRE OVULACION Y TIEMPO DE PRODUCCION SEGUN RESPUESTA DE 229 ESTUDIANTES ENCUESTADAS EN 7 COLEGIOS FEMENINOS DE POPAYAN

PORCENTAJES

\begin{tabular}{|c|c|c|c|c|c|}
\hline & 0 & 25 & 50 & 75 & 100 \\
\hline Correcto & 0 & & & & \\
\hline Incorrecto & & 39.30 & & & \\
\hline Sin contestar & & 51.53 & & & \\
\hline Incompleto & 7.86 & & & & \\
\hline
\end{tabular}

\section{GRAFICA NN 5}

CONCEPTO SOBRE SITIO DEL ORGANISMO EN QUE SE PRODUCE FECUNDACION SEGUN RESPUESTA EN 229 ESTUDIANTES ENCUESTADAS EN 7 COLEGIOS FEMENINOS DE POPAYAN

PORCENTAJES

\begin{tabular}{|c|c|c|c|c|c|}
\hline & 0 & 25 & 50 & 75 & 100 \\
\hline Correcto & 5.76 & & & & \\
\hline Incorrecto & & & & & \\
\hline Sin contestar & & & & & \\
\hline
\end{tabular}

\section{CUADRO NN 16}

CONCEPTO SOBRE SITIO DEL ORGANISMO EN QUE SE PRODUCE FECUNDACION SEGUN RESPUESTAS DE 229 ESTUDIANTES ENCUESTADAS EN 7 COLEGIOS FEMENINOS DE POPAYAN

\begin{tabular}{lrr}
\hline $\begin{array}{l}\text { Conceplo sobre sitio } \\
\text { de fecundación }\end{array}$ & Número & Porcentaje \\
\hline Correcto & 3 & 5.76 \\
Incorrecto & 178 & 77.73 \\
Sin contestar & 46 & 16.50 \\
TOTAL & 229 & 100.00 \\
\hline
\end{tabular}

El comentario del cuadro anterior reafirma en forma amplia los porcentajes aquí observados.

\section{CUADRO NN 17}

CONCEPTO SOBRE BARBA, BIGOTE Y VOZ RONCA EN LAA MUJER SEGUN RESPUESTA DE 229 ESTUDIANTES ENCUESTADAS EN 7 COLEGIOS FEMENINOS DE POPAYAN

\begin{tabular}{lrr}
\hline $\begin{array}{l}\text { Barba, bigote } \\
\text { y voz ronca }\end{array}$ & Número & Porcentaje \\
\hline Correcto & 62 & 27.07 \\
Incorrecto & 103 & 44.98 \\
Sin contestar & 64 & 27.75 \\
\cline { 2 - 3 } TOTAL & 229 & 100.00 \\
\hline
\end{tabular}

Es de notar que solo una cuarta parte tiene un concepto claro sobre este conocimiento rudimentario en relación con el comportamiento hormonal de la mujer.

\section{Discusión}

El análisis de los datos antes mencionados nos demuestran el (Cuadro No 2) que a pesar del alto grado de instrucción de las encuestadas - $59 \%$ cursan último año de bachillerato- manifiestan un desconocimiento absoluto del proceso fisiológico del ciclo menstrual. Esta ignorancia influencia- 
CUADRO № 18

TEMAS ESCOGIDOS POR LAS ENCUESTADAS PARA SER TRATADOS EN CURSOS DE EDUCACION SEXUAL DE POPAYAN

\begin{tabular}{clrr}
\hline No de Orden & Temas Escogidos & Número & Porcentaje \\
\hline 1 & Ovulación, fecundación, embarazo y parto. & 92 & 23.3 \\
2 & Explicación científica del acto sexual. & 77 & 19.5 \\
3 & Fisiología masculina y femenina. & 48 & 12.2 \\
4 & Ciclo menstrual. & 44 & 11.1 \\
5 & Enfermedades venéreas. & 33 & 8.4 \\
6 & Preparación al matrimonio & 21 & 5.3 \\
7 & Iniciación a la vida sexual. & 19 & 4.8 \\
8 & Control de la natalidad. & 16 & 4.0 \\
9 & Sicología masculina y femenina. & 13 & 3.3 \\
10 & Homosexualismo. & 11 & 2.8 \\
11 & Principales enfermedades genitales de la mujer. & 8 & 2.0 \\
12 & Desarmonía matrimonial. & 7 & 1.8 \\
13 & Amor libre, pro y contra. & 6 & 1.5 \\
\hline
\end{tabular}

NOTA: Hay un número de temas mayor que el número de encuestadas porque algunas anotan dos temas. - Esta simple pregunta dio base del temario de cursillos que se realizaron posteriormente.

da en forma notoria por la costumbre y la tradición se han convertido en una serie de prácticas que menoscaban la salud de la mujer.

Es sabido que mientras más atrasados sean los pueblos, mayor es el cúmulo de creencias absurdas que se forjan en la imaginación buscando con ello satisfacer el anhelo común de encontrarle explicación a todo, y remedio a lo que salga de la normalidad. Es antigua la noción de que la mujer que menstrúa es impura, inmunda y contaminante en su persona y que la catenemia es la primera herencia de la mujer moderna, concepto compartido con la religión. Así el Cristianismo en el Levítico XV 19 trae esta cita: "la mujer que tiene flujo de sangre en su cuerpo permanecerá en su impureza por espacio de 7 días y quien la tocare será impuro..."

En la edad media el cristianismo creyendo aún en la impureza de la mujer en el período menstrual impedían que ellas acudieran a la Iglesia
- tomaran la comunión. Corroborando lo anterior el Rito Griego moderno las priva de la comunión y se les prohibe besar cuadros religiosos.

El Islamismo promulgó ideas semejantes y Mahoma afirma en el Corán (Surah 11-222): "Así, manteneos aparte de las mujeres durante su menstruación y no os acerquéis a ellas hasta que se hayan purificado".

Entre los filósofos Aristóteles afirma: "la mirada de una mujer en menstruación despule el espejo, y la siguiente persona que se mire en él queda embrujada".

Las doctrinas hipocráticas la consideraron como una purgación menstrual o como una válvula de seguridad, concepto que con la dominación mora llegó a España y posteriormente a nuestras tierras a través de los Conquistadores y sus acompañantes, según esta creencia por medio de esta función se libra de cuantos humores y males se hayan acumulado en el cuerpo, y que su supresión si no indica embarazo produce enfermedad. 
Las creencias a través del país muestran: en Antioquia el perro cazador pierde su fino olfato si ha olido sangre menstrual.

Entre los guajiros los médicos de la tribu no pueden efectuar los actos ceremoniosos de curación durante el período menstrual, porque el enfermo en lugar de mejorar empeorará.

Estudios en el barrio Siloé de Cali demuestran cómo algunas mujeres creen que la suspensión de la menstruación produce enfermedades como lepra, locuras y acné.

En el Departamento del Cauca existe la creencia de que las mujeres en el período catamenial no pueden "amasar pan" porque éste no levantará, además, en algunas zonas rurales se prohibe la visita de las mujeres menstruantes a las puérperas porque causa "pujos" en los niños y "mal de ojo".

También se le atribuyen poderes curativos, así en Córdoba con la sangre menstruante de doncella, se curan las verrugas escolares, el acné vulgar y los barros.

En el Bajo Cauca se usa contra los dolores causados por la picadura de la raya.

Los ejemplos antes mencionados antiguos y recientes señalan la perpetuación de la idea de que la mujer sobre todo en el período catamenial está poseída por poderes sobrenaturales, sería una semidiosa o demonio, una sacerdotisa o bruja.

Menstruación y Actividades: (Cuadro № 5).

Según creencias bien arraigadas, es prohibido hacer cualquier ejercicio que pueda suspender la menstruación, porque esto "eleva la sangre" 0 "se sube a la cabeza" y pueden presen- tarse hemorragias nasales o cerebrales, para otras, el ejercicio en cambio aumenta la hemorragia poniendo en peligro la salud.

Menstruación y Alimentos: (Cuadros Nos. 6 y 7 ).

Los tabúes alimenticios también son frecuentes y es así como el comer frutas especialmente ácidas es, en regiones de nuestro país, prohibido a las menstruantes porque impera la creencia de que "aguan" o licúan la sangre produciendo por consiguiente un flujo más fuerte con mal olor y color. La leche participa de las anteriores creencias pues su color blanco origina flujos vaginales del mismo color y dá mal olor a la menstruación.

Así mismo es común la creencia de que no se pueden tomar medicamentos y las mujeres sin excepción, preguntan en esta época si las drogas se oponen a ella. $8)$.

Menstruación y Baño: (Cuadro №

En muchas regiones aconsejan evitar el baño, especialmente con agua fría porque puede alterar la salud de la mujer ocasionándole enfermedades de índole diversa y porque la acción del írío, sobre el cuerpo que en su concepto está con su temperatura más alta que la acostumbrada y con los poros abiertos, provoca la suspensión de la menstruación o la salida de sangre por otras cavidades.

Menstruación y Relaciones Sexuales: (Cuadro № 10).

La noción de que las relaciones sexuales durante la menstruación son perjudiciales o peligrosas se encuentra ya consignada en el Antiguo Testamento: Según los antiguos hebreos la cópula durante este período resultaría en un "niño escrofuloso, débil 
mental, epiléptico o loco". En la mitología romana la deformidad de Vulcano se atribuye a relaciones sexuales entre Júpiter y Juno durante el período menstrual de esta diosa. De aquí el concepto muy arraigado de que la sangre menstrual no sólo es mágica sino venenosa y capaz de hacer gran daño.

\section{Menstruación y Cólico Menstrual:} (Cuadro No. 12).

El dolor es sufrimiento, molestia del cuerpo y la mente y denota preocupaciones, problemas a menudo castigo. El dolor es necesidad anormal de solicitud o satisfacción en la mujer masoquista, resentimiento en la frustrada ansiedad en la hipocondríaca, culpa en la exigente e introspectiva, o un mecanismo típico de defensa que linda con la esquizofrenia. Tiene componentes físicos y fisiológicos.

Menstruación y Estado Síquico: (Cuadro № 13).

La sociedad considera a la mujer durante la menstruación, irritable, irascible, incluso irracional. Puede quejarse o molestar al esposo o a la familia y descuidar sus deberes sin temores de reproche. Sus acciones son perdonadas por los demás pues comprenden que "ella está con la enfermedad".

\section{"La enfermedad" de la menstrua- ción:}

La pérdida de sangre incluso en intervalos cíclicos se considera que debilita a la mujer, aunque la menstruación consiste principalmente en el desprendimiento del endometrio, la mujer se siente débil, fatigada y letárgica a pesar de que la pérdida sanguínea es mínima y la concentración de hemoglobina permanece estable, la menstruante se considera vuinerable a los resfriados y a las enfermedades respiratorias por consiguiente en estos días usa ropas abrigadas, se protege contra las corrientes de aire y no se moja ni los pies ni la cabeza, así considerada presume incapacidad física por consiguiente la mujer se inhibe de ir a la escuela o al trabajo y se abstiene de cualquier ejercicio físico.

\section{Resumen y Conclusiones}

1. Por encuesta directa se revisan los conocimientos, aptitudes y valores referentes a la menstruación en 229 estudiantes de último año de bachillerato en 7 colegios femeninos de Popayán.

2) A pesar del alto grado de instrucción hay una gran ignorancia en los elementales conocimientos de la fisiología femenina.

3) Se notan numerosas creencias y prácticas en este grupo estudiantil recibidas por tradición de sus antepasados que perjudican notablemente el desarrollo y la conservación de un mejor estado de equilibrio sicosomático.

4) Estas creencias populares y tabúes tradicionales no deben menospreciarse ni ser motivo de ridículo por quienes tienen a su cargo los programas de orientación y educación sexual de sus semejantes.

5) La universidad colombiana no debe ser cómplice de esta ignorancia especialmente en sus programas extramurales, debe impartir con criterio científico conocimientos sobre los procesos que cumple la mujer durante su edad reproductiva.

6) Como consecuencia deben elaborarse programas educativos sobre la vida sexual de la mujer en forma multidisciplinaria en todas las clases sociales a nivel del hogar, primario, 
secundario y universitario respetando los patrones culturales.

\section{Summary and Conclusions}

1. The knowledge, aptitudes and values concerning menstruation in 229 students of last year of highschool in 7 girl schools of Popayán are analyzed.

2. In spite of the high degree of instruction, there is a great ignorance of the elementary knowledge of the feminine physiology.

3. Numerous beliefs and practices are noted in this student group, acquired through tradition of their ancestors, which substantially damage the development and conservation of a better psycho-somatical balance.

4. These popular beliefs and traditional tabus cannot be overlooked nor become a subject of ridicule for those in charge of the sexual orientation and education of their fellowmen.

5. The Colombian university cannot be an accomplice of this ignorance, particularly in its supra-grounds programs which must be developed with scientific criteria and knowledge on the processes accomplished by woman during its reproduction period.

6. Consequently, educational programs on the sexual life of the wo- man must be developed in a multidisciplinary manner for all social classes at a home level and at grade school, high school and university levels, respecting cultural patrons.

\section{BIBLIOGRAFIA}

1 FENICHEL, O.: Psychoanalytic Theory of Neurosis. Norton, New York, 1945.

2 GIDRO-FRANK, L., GORDON, T. Y TAYLOR, H. C., Jr.: Pelvic pain and female identity: Survey of emotional factors in $40 \mathrm{pa}$ tients. Amer. J. Obstet. Gynec. 79: 1184, 1960.

3 NACAR y COLUNGA Sagrada Biblia - Levitico XV - 19 a 35.

4 HATCH, D. L. y HATCH, M. C.: An unhappy family: Some observations on relationship between Calvinist ethic and niterpersonal relations over four generations, Marriage \& Family Living. 24: 213, 1962.

5 GUTIERREZ DE PINEDA VIRGINIA. Organización Social de la Guajira. Revista del Instituto Etnológico Nacional. Vol. 3. 1948.

6 MELODY, G. F.: Behavioral implications of premenstrual tension. Obstet. Gynec. 17: 439, 1961.

7 CORREA HENAO Folclore de la menstruación. Antioquia Médica. Vol. 16 № 9 y 10 1966.

8 Clínicas Obstétricas y Ginecológicas de Norteamérica. III - 1965.

9 GOMEZ ALDEMAR: Creencias y prácticas populares acerca de la menstruación, gestación, parto y recién nacido en el barrio Siloé de Cali. 1960. Tesis de Grado. 